\title{
LA PROFESIONALIZACIÓN DEL RECURSO HUMANO POLICIAL. ASPECTOS CURRICULARES QUE SE DEBEN CONTEMPLAR
}

\author{
María Gabriela Arce Navarro \\ Estudiante de la Maestría en Planificación Curricular \\ Universidad de Costa Rica \\ San José, Costa Rica
}

Recibido: 27-XI-2007 • Aceptado 11-III-2008 • Corregido 15-IV-2008

\begin{abstract}
Resumen: Este artículo desarrolla una propuesta de organización curricular que garantice el alcance de los objetivos, metas y funciones encomendadas a la Escuela Nacional de Policía, entidad a la que le ha sido conferida la potestad para formar y capacitar a las personas encargadas de velar por la seguridad ciudadana de nuestro país. La propuesta considera elementos aún no establecidos por este centro educativo, aportando avances curriculares significativos para la futura implementación del currículo. Por otro lado, describe los componentes que caracterizan el actual currículo de la Escuela, develando aspectos relevantes sobre la formación y capacitación policial, así como la apertura de esta Institución a los enfoques por competencias laborales, campo en el que incursionó con la implementación del actual Curso Básico Policial por Competencias Laborales.
\end{abstract}

Palabras clave: Formación y capacitación policial, profesionalización policial, currículo policial, competencias laborales.
"La formación es, en la policía, factor de cambio y adaptación permanente a las exigencias sociales, debe ser trazadora de horizontes profesionales adelantándose al futuro".

Carlos Canelo

\section{Introducción}

Durante los últimos lustros, han ocurrido cambios significativos en la profesionalización de la fuerza pública, cambios que están lejos del conocimiento y comprensión de algunos ciudadanos y que han surgido del esfuerzo e interés de personas cercanas al servicio público de la seguridad ciudadana.

La creación de la Ley General de Policía $\mathrm{N}^{\circ} 7410$ en mayo de 1994, es un ejemplo de voluntad y de confianza en un servicio estatal que debe estar en un primer orden, dada la prioridad que tiene en garantizar el orden público, asegurando la tranquilidad y el libre disfrute de las libertades públicas. Sus reglamentos y 
Abstract: This article develops a proposal of curricular organization which guarantees the accomplishment of the objectives, goals and functions entrusted to the National Police School, organization chartered to form and train the people in charge of the citizen security of our country. The proposal considers elements not established by this educative center yet, contributing with significant curricular advances for the future implementation. On the other hand, it describes the components that characterize present curriculum of the School, revealing excellent aspects on the formation and police qualification, as well as the opening of this Institution to the labor skill approach which renewed the current Police Basic Course by Job Skills.

Key words: Formation and police qualification, police professional, police officer curriculum, job skills. artículos vinculantes permitieron dar un salto a la oportunidad de hacer del servicio policial un trabajo dignificante, valioso y profesional.

Si bien, ser policía es pertenecer a una profesión que ha tenido un gran auge y desarrollo en otras partes del mundo (Sotelo, 1989), en nuestra sociedad se han hecho también esfuerzos por profesionalizar este servicio, ya iniciados con la incorporación de la Carrera Policial, tema inmerso en la Ley 7410, propiciando la educación formal entre sus agremiados y la motivación por desarrollarse y crecer profesionalmente en este campo.

Las potestades para el ejercicio y desarrollo de estas capacidades, le han sido conferidas a la Escuela Nacional de Policía, mediante la normativa que regula los principios fundamentales de la capacitación y formación policial, mencionados por la Ley General de Policía, en su Capítulo $\mathrm{X}$, artículo 94.- Criterios, el cual reza lo siguiente:

El adiestramiento y la capacitación policial se fundamentarán en los siguientes criterios:

a) Tendrán carácter profesional y permanente.

b) Serán convalidados por el Ministerio de Educación.

c) No tendrán carácter militar, y en consecuencia, su orientación será civilista, democrática y defensora de los derechos humanos. (Costa Rica. [Leyes y Decretos]. (1994, Artículo 94. Criterios, parr. 1, 2, 3 y 4).

Con base en este marco de referencia, la Escuela Nacional de Policía establece su propia misión y visión, dentro de las cuales se observa la finalidad de contribuir con la sociedad costarricense, al formar a una persona acorde con las necesidades de seguridad de los habitantes. En ellas se visualizan también el espíritu de servicio, interiorizando los principios civilistas, democráticos y de respeto a los derechos humanos. Desde esta perspectiva, se deduce que el Ministerio de Seguridad Pública cree en la profesionalización del personal policial, al considerar este recurso como el 
elemento más importante para desarrollar las estrategias de seguridad a través de una formación que se ajuste a las políticas y doctrinas planteadas.

Así las cosas, reza el siguiente principio de la Doctrina de Seguridad del Ministerio de Seguridad Pública en el considerando No. 8 del decreto No. 32117-SP.

h) En la profesionalización de la policía con base en esquemas que consideren el recurso humano como el elemento más importante para desarrollar las estrategias de seguridad, elegidos bajo un perfil de policía que se ajuste a las tradiciones pacifistas, humanistas, y civilistas del ser costarricense y formado bajo los principios y políticas doctrinarias planteadas (Poder Ejecutivo. Decretos No $32177-$ SP. , 2005, p. 1).

No se pueden dejar de lado los aportes que otras instituciones estatales han realizado como estrategia para promover la profesionalización de los funcionarios policiales, en cuyo caso, la Universidad Estatal a Distancia, mediante la suscripción de un Convenio Marco de Cooperación con el Ministerio de Seguridad Pública en 1997, creó el programa de Diplomado en Ciencias Policiales, otorgándole la oportunidad a este personal de ingresar a un nivel de la Educación Superior. Dicho programa ha estado en vigencia desde el 2001.

Por otro lado, los esfuerzos del Instituto Nacional de Aprendizaje en propiciar los enfoques por competencias laborales dentro de los currícula de la Escuela, han sido significativos. De la experiencia obtenida mediante el trabajo conjunto entre ambas instituciones, se logró un primer acercamiento para la aplicabilidad de dichos enfoques en la elaboración no solo de los diseños curriculares, sino también en la definición de perfiles por competencias laborales. Su contribución quedó demostrada, con el desarrollo del primer estudio que identificaría las necesidades y requerimientos específicos de la primera figura profesional: "Agente de Policía" (denominada así por aquella institución educativa).

Como se observa, propiciar la profesionalización mediante la construcción de estrategias que coadyuven en el fortalecimiento de los conocimientos y capacidades, tanto aptitudinales como actitudinales, ha sido una tarea integrada de estos sectores del país, los cuales desde diferentes ópticas han colaborado por enriquecer los procesos formativos de esta población.

La estabilidad de una sociedad conduce al bienestar en los ciudadanos, y este bienestar se relaciona con el grado de seguridad que disfruta la población civil. De igual forma, el grado de satisfacción que se tenga con respecto a la seguridad dependerá, entre otros, de la preparación con que cuente la persona encargada de ejercer el servicio estatal de la seguridad ciudadana (Canelo y Martín, s.f.), preparación que se visualizará en las capacidades que demuestre durante la realización de determinadas tareas, funciones y actividades que la ley le encomiende.

Desde el momento de creación del Ministerio de Seguridad Pública y posteriormente, de la Escuela Nacional de Policía hasta la fecha, el quehacer policial ha venido desarrollando diferentes formas de currícula, adaptadas cada una al contexto social, nacional e internacional del momento, cuyos procesos de transformación han generado conflictos de intereses, en su formulación, elaboración y desarrollo, siendo que, existieron y existen fuerzas políticas y económicas que imperan, desarrollando presiones que calan en la configuración de los currícula, en sus contenidos y en sus métodos para desarrollarlo (Gimeno, 1998). La formación y capacitación policial de nuestro país, no escapa a esta realidad.

Actualmente, la Escuela Nacional de Policía no tiene un currículo adecuado para formar a las personas encargadas de velar por la seguridad ciudadana de nuestro país. Buscar las razones, no es la cuestión prioritaria en este discurso, pero sí resaltar la existencia de una brecha entre los propósitos de la Escuela y la práctica que se desarrolla. 
La profesionalización de los cuerpos policiales del país no es una tarea fácil de cumplir. Implica la vinculación de varios recursos, que permitan el alcance de sus metas y objetivos institucionales, entre los que destacan: el recurso humano, el recurso material, financiero, y un diseño curricular acorde con las necesidades de la población policial, así como con las demandas de seguridad manifestadas por los ciudadanos.

Con este marco referencial, surge la interrogante por determinar ¿cuáles son los aspectos curriculares que se deben contemplar, para profesionalizar los cuerpos policiales del país? Y de los que mantiene actualmente, ¿cuáles ayudan a la Institución en la satisfacción de las metas, objetivos y necesidades de aprendizaje de su población meta?

Considerando lo anterior, se persigue como propósito realizar una descripción de lo que ha sido la formación policial en nuestro país, desde la perspectiva de su organización curricular, de manera que se puedan identificar los elementos curriculares que le caracterizan.

Se recomendará algunos cambios que se deben aplicar para fortalecer el programa de formación, como una estrategia para el alcance de una política de profesionalización dirigida a la población meta, el Ministerio de Seguridad Pública y la sociedad costarricense.

Para responder al propósito de este trabajo se abordan dos grandes temáticas, una relacionada con los antecedentes de la formación policial la cual está circunscrita en el contexto histórico de la Escuela Nacional de Policía, con la presentación al lector de una descripción actualizada sobre el proceso educativo que desarrolla este centro de formación, para cerrar con los fundamentos curriculares para la formación policial, tales como los Componentes del Currículo, la Concepción de Educación, la Concepción de Currículo, las Bases Curriculares y el Modelo del Diseño Curricular, siendo estos elementos para una eventual reforma curricular.

\section{La formación en la Escuela Nacional de Policía}

El tema de la formación, tanto académica como profesional de los funcionarios que conforman la Fuerza Pública, ha sido parte de la historia pasada y reciente del Ministerio de Seguridad Pública.

Para el año 1849, se establece la institución militar que, apenas si abrigaba a un pequeño grupo de hombres, quienes no tenían una verdadera formación militar y cuyos nombramientos, en los niveles jerárquicos superiores se realizaban atendiendo criterios políticos más que a nociones técnicas o profesionales por lo insipiente y casi innecesaria institución para la bucólica Costa Rica de mediados del siglo XIX.

Bajo esta lógica, era común que a los militares se les asignaran ciertas funciones de orden civil para las que no habían recibido capacitación alguna.

Los hechos históricos de 1949, particularmente con la culminación de la Guerra Civil, generan una serie de acontecimientos y medidas tendientes al debilitamiento del ejército a tal punto que en 1949, y según lo establecido en el Artículo 12 de la Constitución Política donde se proscribe el Ejército como institución permanente, se crea una Guardia Nacional encargada de velar por la seguridad de los habitantes y por el resguardo del orden público.

$\mathrm{Al}$ sustituirse el Ministerio de Guerra por el Ministerio de Seguridad Pública, la entidad militar encargada de la capacitación de los antiguos soldados pasa a capacitar a los miembros del nuevo Ministerio.

En dicho proceso se le cambia el nombre que se le había dado en épocas de la institución castrense, Escuela Militar de Guadalupe, para pasar a denominarse Escuela Cívico Militar, ubicada en Guadalupe, San José y adscrita al Ministerio de la Presidencia, siendo la primera institución de índole policial en donde se capacita a los funcionarios de la Guardia 
Civil (nombre adoptado tal y como lo concebía la normativa española). Las instalaciones de esta Academia fueron donadas al Ministerio de Educación Pública para la instalación del Colegio Napoleón Quesada.

$\mathrm{Al}$ quedarse sin instalaciones, los miembros de la Guardia Civil, adscritos a Seguridad Pública, deberán seguirse capacitando en la Escuela de Policía del Ministerio de Gobernación y Policía, situada para entonces en la provincia de Cartago, de donde sale en 1965 transformándose luego en la Escuela Civil del Ministerio de Seguridad Pública.

Esta Escuela de la Guardia Civil durante unos meses debe buscar nuevas instalaciones para cumplir con su cometido. Las clases se empiezan a impartir en un lugar facilitado por el aeropuerto de El Coco, hoy Aeropuerto Internacional Juan Santamaría.

En 1965, durante la administración del presidente Orlich, se funda en el país la Escuela Nacional de Policía, que tiene como objetivo principal brindar capacitación a los cuerpos de policía. A partir de 1970, durante la administración Figueres Ferrer, mediante decreto 1116-P del 27 de julio del mismo año, se le da el nombre de Academia Nacional de Policía Francisco J. Orlich B., como un homenaje al Ex-presidente costarricense por su aporte en beneficio de la Institución Policial Costarricense.

En 1985 adquiere la condición de Dirección y se desvincula de la Dirección de la Guardia Civil. Se consolida como una Unidad o Programa Presupuestal adscrito al Ministerio de Seguridad Pública, con el nombre de "Escuela Nacional de Policía Francisco J. Orlich B.”.

Desde su creación la Escuela Nacional de Policía implementa acciones de capacitación y formación policial, con la finalidad de desarrollar las competencias y conocimientos necesarios para el desempeño adecuado del quehacer policial, debiendo atender a la población de las diferentes Unidades Policiales adscritas, en sus diversas necesidades educativas.
De acuerdo con la Ley general de Policía 7410, el Capítulo II Título II, le establece a la Escuela Nacional de Policía, dentro del marco normativo, los atributos y deberes de su funcionalidad, otorgándole, entre otros y el más prioritario, la potestad de ser la única dependencia en el país a cargo de la capacitación y formación policial de todos los cuerpos policiales circunscritos en dicha Ley, a saber: Policía Control de Drogas, Policía Fiscal, Policía de Migración, Reserva de la Fuerza Pública, Fuerza Pública, Policía Escolar y de la Niñez, Programa DARE, por citar algunas.

Desde esta perspectiva, la organización tanto curricular como logística debe estar acorde a las necesidades de la Institución, de manera que coadyuven en el cumplimiento de los deberes encomendados por la precitada ley, permitiendo a su vez el logro de sus metas y objetivos estratégicos.

\subsection{La profesionalización policial y los elementos que la caracterizan}

Ubicado el lector en lo que ha sido la historia de la formación policial de la Escuela Nacional de Policía, entramos en el tema de los elementos que caracterizan la profesionalización policial de nuestro país.

En el actual contexto de cambios y reformas, las organizaciones se ven obligadas a ir transformando su dinámica, de manera que les permita estar acordes a las exigencias que el contexto social les exija. Ante este panorama, la Escuela Nacional de Policía deberá asegurar su posicionamiento como institución educativa, garantizando el hecho de que se puedan llevar a cabo las funciones que le competen.

La definición de estrategias de capacitación y formación para la actualización y perfeccionamiento del recurso humano policial, es una tarea constante. Para ello se deberá planificar, administrar, ejecutar, difundir y evaluar las 
diferentes actividades educativas ofrecidas, que le permitan por un lado, valorar y mejorar los procesos de enseñanza que se mantienen y por otro, cumplir con su cometido de hacer del servicio policial un servicio profesional.

El ejercicio de la actividad policial constituye una profesión, teniendo en cuenta que se trata de una tarea permanente, de dedicación exclusiva, por lo que quienes la desempeñan, deben prepararse adecuadamente hasta conseguir plena idoneidad. La verdadera función policial debe poseer formación técnica y académica en forma integral, que le permita en los distintos niveles y jerarquías desenvolverse de manera profesional, ética con liderazgo y vocación de servicio a la comunidad.

Este servicio profesional, desde la perspectiva de Fernández (2001, Breve recorrido histórico del concepto profesión, parr. 1), "puede definirse como una actividad permanente que sirve de medio de vida y que determina el ingreso a un grupo profesional determinado".

Podemos ver en esta definición que la acepción descrita está muy vinculada a un determinado ámbito laboral, a una determinada actividad productiva y de servicio. Por lo tanto, la profesionalización policial, es una actividad perteneciente a un cuerpo policial, siendo a la vez una representación de un organismo civil y estatal, que tiene la responsabilidad de velar porque se cumpla y respeten tanto la Constitución Política, como las leyes y los Reglamentos que de ella se desprenden, protegiendo la vida humana y la propiedad, para hacer más segura, agradable y cómoda la convivencia social (Sotelo, 1989).

En diferentes contextos, la conceptualización de profesionalización está adquiriendo un creciente uso, en ámbitos como los productivos, de servicios y los educativos, esta terminología se visualiza como un elemento que destaca el recurso humano en las organizaciones.

Para el alcance de las metas que se propone, la profesionalización policial requiere de un proceso educativo continuo, flexible, organizado y dotado de una serie de elementos que permitan su dinamismo. A la vez, para que este proceso educativo conlleve las particularidades anteriores, necesita de una organización curricular que contemple componentes tales como la concepción de educación y el enfoque curricular que adoptará, así como las bases curriculares que sustentarán el currículo, por mencionar algunos.

Si bien esta Institución evidencia la presencia de los elementos mencionados, se hace pertinente caracterizarlos de manera que se conozca sobre la organización curricular que mantiene.

La Escuela Nacional de Policía, como administradora del currículo policial, en forma tácita no ha constituido una concepción educativa propia, que le identifique y le oriente en su quehacer educativo, por lo tanto, debe considerarse de gran importancia el generar la conceptualización de currículo y la concepción educativa que va a adoptar de manera que se visualice la orientación educativa que deben tener los programas de formación y capacitación policial.

En este sentido, esta organización educativa ha adoptado la concepción de currículo como la de un programa de estudios. En sus planes de trabajo se fomenta la formación y la capacitación como ejes motores de su quehacer, en donde los elementos endógenos curriculares toman relevancia, al parecer por considerar que sus diseños solo deben responder a este componente curricular, sin tener una noción de que realiza un desarrollo curricular incluyendo otros de sus componentes.

Por otro lado, se logra visualizar en sus programas de estudio componentes, reguladores, metódicos y multimedios, tal vez no tan definidos como suelen aparecer en los diseños curriculares de otras instancias educativas, pero igual presentes en los diseños que elabora este centro educativo. Por su parte, la ausencia del componente orientador, está calando fuerte en la orientación de los programas que diseña y en 
el establecimiento de los perfiles de salida que requiere, por lo que, se hace pertinente dentro de la propuesta de mejora, considerar con un buen fundamento teórico la definición de este componente, el cual, por su escasa presencia dentro del currículo, interfiere en el comportamiento de los elementos endógenos del mismo.

Como otra particularidad que caracteriza la profesionalización policial, se tiene que, realiza una función social (Canelo y Martín, s.f.), ya que satisface una serie de necesidades de la vida social, contribuyendo directamente en el orden de este contexto, en la medida que sus objetivos operacionales se ajusten a las expectativas reales de aquellos a quienes sirve y protege.

$\mathrm{Al}$ dar una mirada a lo que ha sido la formación policial, como una estrategia para alcanzar la profesionalización de este recurso humano, se puede observar que ha sido un proceso gradual y evolutivo, en cuyo caso, lo que más le ha caracterizado es la función socializadora que ha jugado el currículo (Gimeno, 1998).

Construir sobre la función social que desarrolla el currículo dentro de esta organización educativa no es fácil, máxime si hablamos de una institución cuyo accionar no es precisamente el educativo formal.

Sin embargo, se ha podido identificar que sus currícula reflejan el esquema socializador, formativo y cultural que tiene cualquier institución educativa. En este sentido, la Escuela Nacional de Policía tiene su propio esquema formativo y cultural, enmarcado dentro de una doctrina como lo es la seguridad ciudadana.

Como se demuestra en el apartado anterior, desde mucho antes nuestro país viene contando con los servicios de capacitación policial, no tan regulados como lo tenemos ahora, pero igual de importantes para un contexto histórico diferente al actual, tal y como lo expresa el autor Gimeno (1998) los currícula expresados en ese momento histórico, respondieron a intereses y fuerzas particulares, agrupando el conocimiento dentro de una multiplicidad de fines.
De igual forma, la elaboración de los contenidos a estudiar no contaba con la rigurosidad que actualmente se mantiene, pero sí respondían a determinadas necesidades expresadas por un particular grupo social. En un primer momento, la capacitación y formación policial estaba orientada hacia aspectos militares. Después de los acontecimientos de 1949, dejó de existir la estructura militar que la reguló, por lo que adoptaron perspectivas más civilistas que militares.

En este sentido, la orientación de los temas a estudiar varió y se ajustó a nuevos intereses, esta vez de tipo político. Tal y como lo manifiesta Gimeno (1998), la construcción de un currículo no se puede entender en forma separada de las condiciones reales de su desarrollo, por lo que se debe prestar atención a las prácticas políticas y administrativas, condiciones estructurales, organizativas y materiales que se expresan en su desarrollo.

Comprender el proceso social en donde el currículo de esta Institución se ve afectado por diferentes elementos, es comprender su funcionalidad actual, ya que en este centro educativo, al igual, que otras organizaciones, la sociedad contribuye con el currículo, al ser considerada como fuente que aporta necesidades de formación.

Un dato importante de resaltar es que dentro de los procesos de formación que actualmente mantiene esta Institución, se visualizan claramente tres escalas policiales, las cuales a su vez contemplan tres grados policiales. Desde este panorama, están siendo estructurados los diferentes cursos que desarrolla la Institución, los cuales están distribuidos en tres áreas temáticas, a saber: Área Humanística, Área Técnico Policial y Área Jurídica, cada una de ellas está orientada a fortalecer los conocimientos y destrezas de los funcionarios policiales, de manera que se enriquezca la ejecución de las diversas labores policiales encomendadas.

De igual forma, es perentorio mencionar que el Curso Básico Policial es uno 
de los cursos al que la Escuela Nacional de Policía destina sus esfuerzos y recursos, en virtud de ser el necesario y básico para el desempeño de las labores policiales, siendo que, todo aquel funcionario policial para ser considerado como tal, debe poseer la acreditación de haber aprobado el curso en cuestión.

En este sentido, por ser un curso de iniciación, pretende dotar a los funcionarios policiales de los conocimientos básicos para el desempeño de las labores policiales. Con la aprobación de este curso, el funcionario se asegura de realizar sus labores en función de los requerimientos que el contexto le exige, así como, su estabilidad laboral y el pago de los incentivos económicos que esto conlleva, beneficios otorgados por la mencionada Ley General de Policía No. 7410. Cabe señalar que este curso, cuenta con la aprobación del Ministerio de Educación Pública, entidad que lo tiene reconocido.

Es el curso que más variaciones en su diseño ha sufrido, sus contenidos son constantemente modificados, así como las modalidades para su implementación. Es el curso que más tema de discusión ha fomentado, entre las jerarquías de la Institución, mientras que para algunos actores su duración (1050 horas), es insuficiente para el abordaje adecuado de sus diferentes temáticas, para otros, es mucho el tiempo que se dedica a la formación policial por lo que sus contenidos deben ser reducidos, para que en un menor tiempo posible, los funcionarios estén al servicio de la comunidad.

Dado lo anterior, la Escuela Nacional de Policía doblega sus esfuerzos al incorporar dentro de sus estrategias, las experiencias de otros centros educativos, con la finalidad de satisfacer las necesidades que surgen dentro del contexto del Ministerio de Seguridad Pública.

Es así que, instituciones tales como el Instituto Nacional de Aprendizaje, colaboraron en la reelaboración del diseño curricular del Curso Básico Policial, brindando la asesoría metodológica para la construc- ción de lo que sería el diseño curricular del Curso Básico Policial con un enfoque por Competencias Laborales.

\subsection{El diseño curricular con un enfoque por competencias laborales}

Tal y como se mencionó, la Escuela Nacional de Policía en su búsqueda por dotar a la ciudadanía costarricense de un recurso humano con las capacidades necesarias para el desempeño profesional y eficiente del servicio de la seguridad ciudadana, ha adoptado, dentro de sus programas de estudio del Curso Básico Policial la formación con un enfoque por competencias laborales, diseñando sus contenidos en función de lo que identificaría posteriormente, como los grandes ejes temáticos bajo los cuales se orientará la formación y capacitación policial.

Como un primer momento, el nuevo diseño con un enfoque por competencias laborales, se implementó en el curso de iniciación dirigido a toda aquella persona que ingresa a brindar el servicio de seguridad ciudadana: el Curso Básico Policial. Cabe señalar que la implementación del Curso no pasó por un periodo oportuno de validación y valoración, por lo que fue ejecutado sin conocerse resultados preliminares.

Los cinco grandes temas, bajo los cuales se mantiene esta modalidad, fue el resultado de un estudio de campo en varias direcciones regionales del país, en donde fue observado el funcionario policial en su quehacer cotidiano. La información fue recopilada mediante un instrumento diseñado para tal fin. No podía hacerse de otra manera, ya que, una característica de los enfoques por competencias laborales es que toma en cuenta el contexto en donde se desarrolla la competencia, el cual está constituido por un conjunto de situaciones reales (Cejas, 2005).

Del resultado de dichas investigaciones se desprenden los cinco temas a saber: 
Manipulación de armas de fuego, Técnicas de intervención policial, Ubicaciones policiales, Mantenimiento del orden público y Comunicaciones policiales.

La determinación de estas competencias, no escapan a la realidad de lo que está definido en el contexto social sobre lo que significa una competencia laboral, por mencionar algunas definiciones, Cejas (2005), en el bagaje de acepciones a las que se refiere, menciona que es un conjunto de conocimientos teóricos, habilidades, destrezas y actitudes que son aplicados por el trabajador en el desempeño de una ocupación determinada. Por otro lado, Chávez (1988 citado en Salas, 2005, p. 5) indica que es el resultado de un proceso de integración de habilidades y de conocimientos, saber, saber-hacer, saber-ser, saber-emprender.

Con este marco de referencia, se puede manifestar que las competencias identificadas responden de alguna manera a esta vinculación de términos, ya que vienen a ser lo que el funcionario policial desempeña en la ejecución de un determinado trabajo.

Tal y como se observa, estas competencias están en función de desarrollar ciertas destrezas y habilidades conjugadas con la asimilación de conocimientos que le den el aporte técnico necesario para el eficaz ejercicio de las labores de seguridad ciudadana, cuyo resultado es la formación de personas capaces de aportar y transformar a la sociedad.

Si bien, las competencias descritas, son el reflejo de un saber-hacer, llama la atención que en su definición, no fueron consolidadas aquellas relacionadas con un área muy vinculada a la labor policial: la atención a los habitantes.

En este sentido, Cejas (2005), menciona que una competencia es un sistema de componentes cognitivos, metacognitivos, motivacionales y cualidades de la personalidad, que le permiten a un individuo desenvolverse eficientemente en la vida como persona en todas sus facetas.
Por otro lado, el mismo autor afirma que las competencias deben ser procesos integradores de conocimientos, habilidades, componentes motivacionales, cualidades de la personalidad, componente metacognitivo, valores y actitudes. En otras palabras, este tipo de enfoques debe buscar no solo formar una persona para un determinado trabajo, tarea o función, sino que debe formarlo para la vida.

Se aprecia que la formación con un enfoque por competencias laborales, no se limita a orientar los procesos educativos en función de lo que la persona puede ejecutar para el desempeño de una determinada labor, sino que, rescata los elementos motivacionales y actitudinales que pueden desarrollar en una persona, o sea su saberser. Este elemento de formación es parte fundamental de la función policial. El saber ser mejores policías con calidad humana, no debe dejarse de lado en este importante servicio, debiendo cuidar esta dimensión actitudinal en su trabajo.

La labor policial implica la vinculación de relaciones directas e indirectas con la ciudadanía. Al ser un servicio público, la idea de atención debe estar presente en todas sus actuaciones y en la base de toda actuación policial. Debe estar presente el respeto a la dignidad, la libertad, la integridad física, la igualdad, la vida del ciudadano al que atiende en cualquier momento. Este servicio de atención, debe pretender la protección integral de la persona, protección y amparo en situaciones críticas, mediante la aplicación de medidas oportunas.

Así las cosas, el establecimiento de una base curricular que sirva de fundamento y orientación a sus programas de estudio, es una necesidad latente, por consiguiente en los próximos apartados se presentará un acercamiento para una eventual reforma curricular dentro de la Escuela Nacional de Policía, mediante la cual se pretende orientar y organizar los currícula que desarrolla esta organización educativa. 


\section{Bases para la formación policial}

Anteriormente había sido caracterizado el proceso de la profesionalización policial, exaltando algunos de los elementos que considera la autora más destacable para el presente artículo, sin restarle importancia a otras particularidades que se puedan denotar en este proceso. Establecer las bases para la formación policial, que coadyuven en la profesionalización de este grupo en particular, es el propósito de este apartado.

Actualmente, la sociedad costarricense exige una policía profesionalmente bien preparada, capaz de dar respuestas oportunas, rápidas, eficaces y técnicas ante situaciones de delincuencia, conocedora del contexto social donde realiza el ejercicio de sus labores, y por ende, con las suficientes calidades humanas, como para que le permitan interactuar con sus principales actores: los habitantes de este país.

Dado lo anterior, la formación policial adquiere cierto valor al estar en función de las pretensiones sociales que le imperan, por lo que, es pertinente un modelo de formación acorde a la satisfacción de las necesidades y demandas de los habitantes y de la población meta.

Para el autor Canelo y Martín (s.f.), usualmente los modelos de formación policial son definidos por negociación política. Nuestro país no escapa a esta realidad, ya que en la actualidad, esta intervención en los programas de estudio es latente, con una clara intensión de satisfacer intereses y sensibilidades de ciertos grupos, los cuales tienen una escasa concreción profesional.

Así pues, predominan en los programas de estudio, ideales de formación muy lejos de ser alcanzados, constituidos bajo perfiles sin una definición profesional clara, sin mayor orientación que la propia experiencia policial que sustenta los procesos de enseñanza aprendizaje, dejando de lado los ideales de formación y capaci- tación que persigue la Escuela Nacional de Policía, así como aquellos que requiera la sociedad costarricense.

Es por tanto que, para el alcance de un modelo de formación profesional, deben constituirse las bases curriculares lo suficientemente sólidas que le garanticen, tanto a los habitantes como a sus beneficiarios, una educación acorde a sus necesidades y expectativas laborales.

\subsection{Los componentes del currículo policial}

Dentro de todo modelo curricular se sistematizan los elementos del currículo, elementos que deben estar presentes en el modelo curricular de la Escuela Nacional de Policía. En este sentido, Bolaños y otros (2006) infieren que dentro del currículo se encuentran inmersos una serie de elementos que interactúan entre sí, dichos elementos tienen funciones predominantes dentro del proceso curricular, adquiriendo también una naturaleza única y particular, de acuerdo con el enfoque y concepción curricular que adopte el modelo curricular, que en el caso de la Escuela Nacional de Policía por la naturaleza del quehacer policial, los elementos curriculares que seleccione se verán operacionalizados de acuerdo con la concepción y enfoque curricular seleccionado.

Los elementos curriculares a considerar serán los mencionados por Bolaños y otros (2006), los cuales siguen planteamientos teóricos de Viola Soto, elementos que se reconocen en la obra de Villarini (1996), al caracterizarlos como elementos que componen las fases para la elaboración de un currículo, a saber.

Un modelo formativo policial, en forma general, debe considerar en su formulación los componentes orientadores, mediante los cuales se expresan las grandes finalidades a nivel macro, hacia dónde se desea orientar el conocimiento y lo que se espera que el funcionario policial logre 
mediante las actividades de enseñanza. Este elemento viene a definir los fines y objetivos del programa en sí.

En el caso que nos ocupa, los fines y objetivos de los Programas de estudio de la Escuela Nacional de Policía deben esbozar lo que perseguimos con esta formación, así como la misión, la visión, los objetivos y las políticas de la Institución en particular, y en general del Ministerio de Seguridad Pública, los cuales a su vez responden a nuestro ordenamiento jurídico constitucional.

De igual forma, se hace pertinente para el desarrollo de los modelos formativos policiales, considerar los fines educacionales establecidos por el Ministerio de Educación Pública, definidos en la Ley Fundamental de Educación, instrumento esencial que contiene los principios axiológicos generales de la educación costarricense. Este elemento se encamina hacia la consecución del qué enseñar para alcanzar niveles de profesionalización policial óptimos.

Por otro lado, es oportuno considerar los componentes reguladores, dentro de los cuales se conjugan los componentes que norman el proceso curricular, denominados también como elementos endógenos, en este sentido se tiene entonces que los objetivos constituyen el resultado que se espera alcanzar con la formación y capacitación policial recibida. Al plantear los objetivos, el instructor deberá garantizar que éstos tiendan a fortalecer el desarrollo integral del proceso de aprendizaje. En cuanto a los contenidos, se debe definir como el cuerpo de conocimiento (hechos, datos, conceptos, principios) que se desprenden de las tres áreas de conocimiento, el cual es desarrollado mediante los procesos de enseñanza aprendizaje. El contenido es un medio para ejercitar y desarrollar procesos y habilidades de pensamiento.

La metodología, son las actividades que le proveen experiencias personales de aprendizaje, estas actividades deben ser propiciadas o motivadas por la institución, responden a los intereses, necesidades y expectativas de los estudiantes. El instructor debe estructurar las actividades de enseñanza aprendizaje con el fin de alcanzar los objetivos propuestos en relación con los contenidos seleccionados. Por consiguiente, la metodología para las actividades de formación policial, debe enfatizar en los procesos participativos, organizativos y democráticos. En cuanto a la evaluación es el proceso mediante el cual se puede percibir el logro de los objetivos propuestos y los avances que muestran los alumnos. Mediante la evaluación el instructor debe propiciar verdaderas situaciones evaluativas en las que los estudiantes demuestren la adquisición o dominio de destrezas, habilidades y conocimientos, así como introducir las correcciones oportunas en caso contrario.

No se pueden dejar de lado, los elementos generadores, en donde el papel de la sociedad, los estudiantes y sus instructores dentro de los currícula de este centro educativo, son los portadores del conocimiento, ya que la sociedad costarricense en sí misma es una generadora del currículo policial, al igual que sus estudiantes y sus instructores, quienes a través de sus experiencias, enriquecen el proceso educativo al llevar los conceptos teóricos a prácticas cotidianas.

En cuanto a los componentes activadores o metódicos, los procesos de enseñanza y aprendizaje de la formación policial requieren de la implementación de métodos específicos que estén presentes en sus diferentes fases, desde su planeación hasta su ejecución y evaluación, las experiencias de la población de este centro formativo están condicionadas a escenarios naturales propiciados por las mismas comunidades, con el fin de asimilar lo más parecido posible su realidad operativa. No menos valor tienen los componentes multimedios, que son necesarios dentro de los procesos de enseñanza-aprendizaje policiales. $\mathrm{Al}$ ser recursos de apoyo en la instrucción, pueden enriquecer los procesos de formación, proyectando a los estudiantes distintas situaciones cotidianas que viven las personas encargadas de la seguridad costarricense. 
Dado que esta institución carece de algunos recursos que coadyuven en el desarrollo pleno de los procesos de enseñanza, es útil retomar los escenarios propios y dinámicos que le proporcionan las mismas comunidades donde en un momento determinado desempeñarán sus funciones.

Todos estos componentes entran en juego, a través de las diferentes acciones dinamizadoras del currículo, en donde, cada uno de ellos asume un papel específico y determinante dentro del currículo de esta Institución.

Como complemento a estos componentes, las propuestas de formación policial, deben estar compuestas por un determinado enfoque curricular, mediante el cual se determine la concepción de persona que se pretende formar. Desde esta perspectiva se puede afirmar que los enfoques curriculares son el énfasis teórico que adopta un determinado proceso educativo para caracterizar y organizar internamente los elementos del currículo. El contenido y la forma del currículo deben estar comprometidos con una determinada concepción de persona que será el ideal a formar.

Con este preámbulo de componentes y elementos que se deben observar en la formulación de ofertas curriculares para la formación policial, se propone la siguiente concepción de educación, la cual contempla unidades curriculares que se sugiere sean considerados en el planteamiento de futuros cursos de formación y capacitación policial.

\subsection{Concepción de educación para los programas educativos policiales}

La Escuela Nacional de Policía, se ha convertido en un medio no formal para la educación de funcionarios policiales. Estructura que sigue una directriz específica creada y establecida para su regulación mediante una normativa que regula los principios fundamentales de la capacitación y formación policial, sobre los cuales se ha hecho referencia con anterioridad.

La visión y la misión establecidas por la Escuela Nacional de Policía, surgen de nociones generales que le están predeterminadas en los marcos normativos que la regulan, y por tanto, tiene como finalidad contribuir con la sociedad costarricense, formando a un funcionario policial que responda adecuadamente ante las necesidades de seguridad que reclaman constantemente los habitantes. Este funcionario policial demostrará valores inherentes a la disciplina, integridad moral, y una relación positiva de servicio al ciudadano.

Dado lo anterior el quehacer de la Escuela debe responder a la formación de un funcionario policial, que traduzca sus capacidades, para entender a la prevención como la adopción de políticas tendientes a impedir la comisión de delitos o la aparición de la delincuencia, tomando las acciones necesarias para la elaboración de estrategias que disminuyan el delito y el sentimiento de inseguridad.

Por otro lado, propiciará la formación de una persona que mediante la acción educativa, alcance niveles adecuados en $\mathrm{su}$ desarrollo integral, en estricto apego al respeto de los derechos humanos, a las normativas vigentes, comprometido con el país, con sentido civilista sobre su quehacer diario, en fin un ciudadano útil a la sociedad y a sí mismo.

Los fines educacionales que ha establecido el Ministerio de Educación Pública, promueven la formación de ciudadanos amantes de su patria, con total conocimiento de sus deberes, derechos y libertades fundamentales; dispuestos a conciliar los intereses individuales con los de la comunidad, ciudadanos con la capacidad de contribuir al desenvolvimiento pleno de la personalidad humana, estimulando el desarrollo de la solidaridad y de la comprensión humana, que promueva la crítica, la búsqueda de soluciones a problemas comunales y en general al avance de la sociedad a través del bien común. 
Se pretende que la siguiente propuesta, propicie un modelo formativo policial centrado en el estudiante, en sus necesidades, intereses y problemas. Debe observar al estudiante como un ser activo, dinámico y participativo. La concepción de educación personalizada debe ser completa y coherente, que atienda el desarrollo total y armónico de la persona respetando su autonomía y crecimiento vital.

Para el alcance de estos fines se hace pertinente desarrollar en los procesos de enseñanza aprendizaje policial, el rescate de las experiencias de los educandos, para que sea enriquecido y retroalimentado el conocimiento. La comunicación dentro de estos procesos educativos debe ser fluida, constante e integral, permitiendo la discusión y análisis crítico de los educandos. El estudiante es el actor principal y el instructor policial como orientador del proceso, basado en programas y planes de estudio en el marco del modelo integrador que refleja una estrecha relación entre contenidos y la labor cotidiana de la población.

La educación debe promover los medios necesarios que permitan dar respuesta a intereses individuales y colectivos. $\mathrm{Al}$ respeto por diferentes formas de aprendizaje e inclusive a los ritmos de aprendizaje de cada estudiante, la educación también debe ser concebida como una fuerza vitalizadora, movilizadora, que responda a las necesidades individuales de crecimiento y de realización humana.

En este sentido la educación que propone la Escuela Nacional de Policía, debe caracterizarse por contemplar un proceso de enseñanza aprendizaje armónico, posibilitando los medios para la liberación y desarrollo de cada estudiante. De manera que se le ofrezca al estudiante policial los recursos y las experiencias que contribuyan a su crecimiento personal y profesional.

\subsection{Concepción de currículo}

El currículo de la Escuela Nacional de Policía es una guía para los instructores y personal competente para su desarrollo, es un instrumento útil para orientar la práctica educativa. Por tanto el currículo no puede limitarse solo a enunciar una serie de situaciones, intenciones, principios y orientaciones generales, debe estar lo más cercano posible de la realidad de las aulas, de la realidad operativa policial, de la realidad cotidiana policial, su aporte para los instructores es nula si no tiene este complemento. Siendo que, un acercamiento hacia su conceptualización debe contemplar la anterior premisa.

Quienes administran un centro educativo deben tener claridad en la conceptualización de currículo que han de adoptar (Chen y Vargas, 2007), por otro lado, esta comprensión del concepto de currículo, está relacionada con un dominio en la concepción educativa que ha adoptado la Institución.

La Escuela Nacional de Policía, como administradora del currículo policial, se refiere en su concepción de currículo, como un conjunto de elementos formales, actitudes, valores y creencias (De Alba, 1994), que favorecen y promueven el desarrollo personal y profesional del estudiante.

Dado que la concepción de educación propuesta, pretende el desarrollo integral de sus estudiantes a través de experiencias satisfactorias e integradoras, el currículo se visualiza como una totalidad en donde convergen dos aspectos: la realidad social y la propuesta educativa (Quesada y otros, 2001), de manera que los componentes del diseño curricular que lo integran guarden una relación entre sí.

Esta concepción de currículo no pretende observar al contenido como un conglomerado de fases en donde el contenido está por un lado, los métodos y metodología por otro y desvinculados de los objetivos de aprendizaje. Al contrario, las decisiones que se tomen con respecto a algunos de los componentes, afecta a los demás.

Una concepción de currículo personalizado, tiene como característica la responsabilidad del educando en cuanto al progreso de su aprendizaje, o sea, le permite 
al estudiante aprender por sí mismo, adecuándose a las capacidades de cada estudiante. Por otro lado, está vinculado a un cuerpo de valores universales, a la atención de la persona y la constante búsqueda, para que cada una de esas personas construya su proyecto personal de vida, de manera libre, responsable y auténtica, permitiéndole conocerse así mismo.

Desde esta perspectiva el currículo de la Escuela Nacional de Policía pretende propiciar un desarrollo individual en cada uno de sus estudiantes, fomentando a través de sus procesos de enseñanza, el diálogo abierto entre sus instructores y sus estudiantes, respetando las diferentes formas de aprendizaje de los educandos, prestando un mayor interés en la calidad de lo que se está enseñando.

Esta concepción curricular, Villarini (1996) la denomina como de desarrollo humano, en donde se convierte en centro de atención tanto las experiencias como las necesidades del estudiante, siendo su principal meta, el desarrollo integral del estudiante, en sus dimensiones intelectual, emocional, social, y psicomotora.

$\mathrm{Al}$ ser considerado dentro del currículo del Curso Básico Policial el enfoque por competencias laborales, la Escuela Nacional de Policía, fomenta en el recurso humano policial desarrollar capacidades propias del quehacer de la seguridad ciudadana, dentro de las cuales no solo serán importantes las capacidades de ejecución y conocimiento sino que serán integradas una actitud solidaria con los ciudadanos y sus compañeros, una disposición singular y comprometida con la seguridad ciudadana, en otras palabras, "saber hacer acompañado de querer hacer" (Canelo y Martín, s.f., p. 27).

Aunado a lo anterior, para la Escuela Nacional de Policía las competencias policiales se encuentran definidas en los siguientes aspectos, (Villarini, 1996), de manera que, sean desarrolladas las competencias policiales sobre la reflexión del estudiante, de sus experiencias previas y del contexto donde se ubica.
- Son las capacidades que el policía desarrolla gradual y acumulativamente a lo largo del proceso de formación.

- Son las características que el policía manifiesta en diversas situaciones y escenarios sociales. Moldea su capacidad a los requerimientos del entorno, da respuesta al mismo, de otro modo, desarrolla un saber situacional o contextual.

- Son las capacidades para desempeñar diferentes áreas del quehacer policial de una manera eficiente $y$ adecuada.

- Son las cualidades que la sociedad costarricense estima convenientes en la formación de ese recurso humano.

- Son las capacidades para adquirir los conocimientos y posteriormente, asumir control sobre ellos.

\subsection{Bases curriculares}

Todo currículo se sustenta en principios que aportan diferentes disciplinas bajo las cuales éste se apoya. El fundamento curricular que propiciará un modelo educativo para la Escuela Nacional de Policía, como estrategia para alcanzar un nivel adecuado de profesionalización policial, se enmarcará desde tres perspectivas.

Desde la perspectiva filosófica se pretende que el funcionario policial, sea una persona con altos valores cívicos; en tanto que reconozca su amor por la patria, y por los símbolos cívicos que la representan. Una persona de respeto, en tanto sea capaz de respetarse primero así mismo, para posteriormente, respetar a sus semejantes (familiares, compañeros de trabajo, ciudadanos, etc); de valores morales, dado que la función policial, es la herramienta que garantiza el estado para fortalecer el desarrollo de los habitantes de la nación, en un marco de seguridad, respetando y promoviendo, la conciencia democrática, pacifista, humanista y civilista. Con una identidad propia. Por otro lado, al considerar las potencialidades, 
el estudiante fomenta la exploración, el cambio, la creatividad, la autonomía, la libertad, la responsabilidad, y el diálogo.

Para el desarrollo oportuno de la propuesta se requiere una persona motivada y llena de confianza para actuar, por lo que estas características se alcanzan únicamente en un ambiente de respeto, comprensión y apoyo para los estudiantes.

\section{Desde la perspectiva psicopeda-} gógica el conocimiento que adquiera responderá primero a las necesidades que requiera el desempeño de sus labores, el cual por la definición del enfoque adoptada, se asemeja a un aprendizaje construccionista, en donde, los procesos de aprendizaje se alimentan, con las experiencias cotidianas de los estudiantes, así como las de los funcionarios docentes, permitiendo la inclusión de nuevos y modernos contenidos, obtenidos de la cotidianidad de la labor policial, labor que está inmersa en un contexto social, cambiante, donde surgen nuevos modelos y estrategias delictivas, de manera que el funcionario policial, deba adaptar las nuevas experiencias, convirtiéndolas en nuevas estrategias de capacitación.

Y finalmente, desde la perspectiva cultural social debe resaltarse que el quehacer policial está inmerso dentro de la sociedad. Para la elaboración del currículo de la Escuela Nacional de Policía, el contexto social en el cual deben obtenerse insumos importantes, vienen a ser las mismas comunidades donde el funcionario policial desempeña un determinado trabajo policial, pues las necesidades de seguridad no son las mismas en una comunidad que en otra. Dado lo anterior, los diferentes actores que subyacen en este contexto, interfieren relativamente en su desempeño, tales como instituciones presentes dentro de la comunidad, fuerzas vivas de la localidad, entre otras. Por otro lado, la comunidad está inmersa en un contexto social, más amplio, el cual es nuestra propia sociedad costarricense, dotada de una diversidad de culturas y costumbres, que a su vez está inmersa en un contexto regional, en donde se citan un sinnúmero de actores que hacen de la zona una región particular. Es por ende recalcar que el actuar delictivo está presente, en todos los países, regiones y zonas geográficas, en donde se da el fenómeno de la interacción entre los delitos internacionales con los asentados en el país, fortaleciéndose cada día más, una delincuencia especializada de carácter transnacional.

\subsection{El diseño de modelo curricular policial}

Se entenderá como diseño curricular a la elaboración de un plan que se traza con la finalidad de que, la ejecución de la actividad educativa, siga una serie de pasos determinados por un hilo conductor, secuenciados en forma lógica, que culminen con la satisfacción de un proceso de enseñanza-aprendizaje predeterminado.

Por tanto, se considera oportuno desarrollar un modelo de diseño curricular de corte deliberativo y práctico, de manera que en su construcción permita la participación de todos sus actores, entre ellos, el instructor, quien mediante sus experiencias cotidianas plantee un diseño con un lenguaje y en un contexto no ajeno a la realidad educativa.

Desde esta perspectiva, se persigue que la Escuela Nacional de Policía, considere la participación de todas aquellas personas que están relacionadas con el currículo policial, entre ellos, el mismo instructor, quien a través de su experiencia y práctica policial, le han permitido adquirir un conocimiento importante de transmitir a los estudiantes de este centro educativo, considerándose un insumo fundamental en los procesos de diseño curricular.

Por otro lado, posibilita el empleo de su conocimiento práctico para la toma de decisiones sobre lo que enseñan y cómo lo enseñan, reflexionando sobre la práctica operativa policial, para posteriormente dar alternativas en su futuro proceder. 
De igual forma, este tipo de modelo permite la convergencia e integración pragmática, de diferentes teorías, visiones, enfoques y procedimientos, las cuales salen a relucir, mediante el debate y la deliberación constante de todos los participantes en la construcción del diseño curricular policial. De esta forma, no existirá manipulación ni influencia alguna, sobre los contenidos de los cursos que se implementen en este centro de formación.

El quehacer policial implica una práctica operativa rigurosa, disciplinada y eficiente, por lo que su enseñanza, debe reflejar lo más cercana posible, cómo es la realidad en donde se opera policialmente y dado que las realidades sociales son dinámicas por naturaleza, las instituciones estatales deben estar acorde a las exigencias actuales de la sociedad, en este sentido, la práctica policial debe concebirse como un proceso de investigación educativa constante y periódica, de manera que, sean transmitidos a los estudiantes, conocimientos prácticos actualizados. Renovándolos de acuerdo con la necesidad operativa que surja del dinamismo de la sociedad.

Este tipo de diseño curricular se asemeja a los intereses que persigue la fundamentación del enfoque curricular, en tanto permite la deliberación de sus diseñadores, cediendo espacio a la toma de decisiones de todos sus actores.

La planificación curricular es una empresa en donde el diseñador, de manera objetiva, propone los medios necesarios para producir los resultados de aprendizaje deseados. En este proceso no hay espacios para las valoraciones personales, solo la eficiencia para la consecución de los objetivos planteados.

\subsection{Planteamiento del perfil policial}

Con este planteamiento, se busca consolidar un perfil que responda a las capacidades y conocimientos que responderán al Curso Básico Policial con un enfoque por
Competencias Laborales, como un primer acercamiento, para futuros perfiles que requiere la Institución. En este sentido, los estudiantes y las estudiantes al terminar el curso demostrarán las siguientes capacidades.

- $\quad$ Conocer, comprender e interpretar, la realidad sociocultural de su entorno.

- Poseer un dominio en el uso de los procedimientos y las técnicas que permitan una adecuada intervención policial.

- Aplicar las medidas de seguridad, así como los elementos jurídicos que norman y regulan el uso y la manipulación de las armas de fuego autorizadas por la ley General de Policía.

- Usar los equipos de radio comunicación policial, de manera que se facilite la comunicación entre las diferentes unidades policiales.

- Controlar y mantener el orden público y la seguridad en concentraciones y manifestaciones de los habitantes.

- $\quad$ Ser hábil para ubicarse en diferentes contextos territoriales del país.

- Identificarse con los valores éticos, morales y cívicos que distinguen los cuerpos de policía.

- Mantener buenas relaciones interpersonales en su ámbito de trabajo con compañeros y con los usuarios del servicio.

\section{Reflexión final}

Este no es un análisis acabado, apenas inicia; en próximos artículos se pueden abordar otros elementos más concretos cuyas particularidades colaboren en la definición de un proceso de formación policial adaptado a la realidad policial y que por ende, contribuya en el fortalecimiento de su profesionalización.

Un análisis de lo que está pasando en el desarrollo del currículo de la Escuela Nacional de Policía y proponer la 
incorporación de algunos aspectos curriculares dentro de sus diseños curriculares acorde con las características propias que evidencia la Institución y los distintos actores en el proceso educativo, podría ser un primer paso en la consecución de una sistematización de las labores de enseñanza aprendizaje de este campo profesional.

Pero la tarea no debe quedar aquí, la búsqueda de mecanismos y estrategias que fortalezcan la profesionalización de los cuerpos policiales del país, debe ser tema de interés en todos los ciudadanos costarricenses, comprometidos con uno de los pilares de nuestra sociedad, el cual es la seguridad ciudadana.

Como es evidente en la lectura llevada a cabo, parte del alcance de un nivel de profesionalización para los cuerpos de policía de nuestro país está en los procesos de formación y capacitación que se implementan, específicamente los desarrollados en la Escuela Nacional de Policía, que como queda claro, ha sido la designada en la consecución de esta importante labor.

Las mejoras sugeridas para rediseñar su currículo vigente, es un aporte de la autora que, como ciudadana, veo la necesidad de contribuir desde mi ámbito profesional, en el fortalecimiento de una de mis necesidades más apremiantes, mi propia seguridad, la de mi familia y la de todos mis conciudadanos costarricenses.

Por tanto, el discurso presentado no solo fue reflexivo y serio, sino que ofreció una alternativa curricular como estrategia para una profesionalización policial acorde a las necesidades institucionales y de la sociedad costarricense, propuesta que puede ser mejorada, de acuerdo a las exigencias que evidencia nuestro contexto social y porqué no, de su evolución. Como se ha podido observar, los primeros intentos por lograr una profesionalización policial congruente, han constituido un proceso de maduración, mediante el aprendizaje del ensayo y el error.

Los esfuerzos por elevar las capacidades de los funcionarios policiales, deben ser constantes, abiertas y flexibles, cuyo único objetivo es lograr un recurso humano policial debidamente formado y capacitado, con las competencias que requiere la atención y resguardo de nuestra seguridad. Una de nuestras principales necesidades básicas.

Como se mencionó anteriormente, el presente discurso, es solo un comienzo, un acercamiento a un quehacer que es del interés de todos los habitantes y las habitantes. Las tareas que quedan pendientes, serán en un futuro el aporte de otras personas para enaltecer, dignificar y profesionalizar el cuerpo policial de Costa Rica y por ende, la seguridad ciudadana.

\section{Referencias bibliográficas}

Bolaños, G. y Molina Z. (2006). Introducción al currículo. San José: EUNED.

Bolívar, A. (2000). Modelos de diseño curricular de corte crítico y posmoderno: descripción y balance. En: J. M. Escudero (Coord.), Diseño, desarrollo e investigación del currículo (pp. 145164). Madrid: Ed. Síntesis.

Canelo, C. y Martín, Q. (s.f.). Base Científica de la Formación Policial. España: Centro de Formación de Ávila, Investigación Publicada.

Cejas, E. (2005). Los fundamentos del Diseño Curricular por Competencias Laborales. La Habana, Cuba: ISPETP. Mimeografiado.

Costa Rica. [Leyes y Decretos]. (1994). Ley General de Policía. Capítulo $X$ : Adiestramiento y Capacitación. Artículo 94.- Criterios. [San José, C. R.]: Publicaciones Jurídicas. Extraído el 29 de octubre del 2007 de http:// www.poder-judicial.go.cr/salatercera/ leyes/leypenal/leydepolic\%C3\%ADa. $\underline{\mathrm{htm}}$ 
Chen, E. y Vargas, I. (2007). Administración de recursos institucionales en centros educativos. Heredia, C. R.: EUNA.

De Alba, A. (1994). Currículo: Crisis, mito $y$ perspectivas. México: Universidad Nacional Autónoma de México.

Fernández, J. (2001). Elementos que consolidan el concepto de profesión. Notas para su reflexión. Revista Electrónica de Investigación Educativa, 2(3). Extraído el 3, marzo de 2007 de http://www.redie.ens.uabc.mx/ vol3no2/contenido-fernandez.html

Gimeno, J. (1998). Aproximación al concepto de curriculum. [Capítulo I] y La selección cultural del curriculum [capítulo II]. En J. Gimeno (Aut.) El curriculum: una reflexión sobre la práctica $\left(7^{\circ}\right.$ ed., Cap. I, pp. 13-64 y Cap. II, pp.65-105). Madrid: Ediciones Morata, S. L. Capítulo 1 y 2.

Ministerio de Seguridad Pública, Costa Rica. (s.f.). Escuela Nacional de Policía. Historia. Extraído el lunes 10 de enero del 2005 de http://www.msp. go.cr/escuela_policia/historia.html

Poder Ejecutivo. Decretos $\mathrm{N}^{\mathrm{o}}$ 32177-SP. (2005). Reglamento de Organización del Ministerio de Seguridad Pública. Considerando $\mathrm{N}^{\circ}$ 8. Alcance $N^{\circ} 1$ a la Gaceta $N^{o}$ 6, Diario Oficial, Año CXXVII. La Uruca, San José, Costa Rica, lunes 10 de enero del 2005, p. 1. Extraído el 29 de octubre del 2007 de http://historico.gaceta.go.cr/2008/01/ ALCA1_09_01_2008.pdf

Quesada, M., Cedeño, M. y Zamora, J. (2001). El diseño curricular en los planes de estudio: aspectos teóricos y guía metodológica. Heredia Costa Rica: EUNA.

Rico, J. y Salas, L. (1988). Inseguridad Ciudadana y Policía. Madrid: Tecnos, S.A.

Rojas, C.(s.f.).Diagnósticodelplancurricular de la Escuela Académica Profesional de Obstetricia de la Facultad de Medicina de la Universidad Nacional Mayor de San Marcos y sus efectos en el perfil profesional. Extraído el 29 de octubre 2007 de http://sisbib.unmsm. edu.pe/Bibvirtual/Tesis/Human/ Rojas_E/Cap2.htm

Salas, W. A. (2005, 29 de Setiembre). Formación por competencias en Educación Superior. Una aproximación conceptual a propósito del caso colombiano. Revista Iberoamericana de Educación, 36, 1-11. Extraído el 29 de octubre de 2007 de http://www. rieoei.org/1036salas.htm

Sotelo, L. F. (1989). Policía Profesional. México: Editorial Limusa, S. A.

Villarini, A. (1996). El currículo orientado al desarrollo humano integral. Río Piedra, Puerto Rico: Biblioteca del Pensamiento Crítico. 